\title{
THE USE OF PRIVACY-PROTECTED COMPUTER VISION TO MEASURE THE QUALITY OF HEALTHCARE WORKER HAND HYGIENE
}

\author{
Running Title: Hand hygiene and computer vision \\ Word Count: Structured abstract -245 , Body text -2477
}

\begin{abstract}
Objectives. (i) To demonstrate the feasibility of automated, direct observation and collection of hand hygiene data, (ii) to develop computer visual methods capable of reporting compliance with moment 1 (the performance of hand hygiene before touching a patient), (iii) to report the diagnostic accuracy of automated, direct observation of moment 1.

Design. Observation of simulated hand hygiene encounters between a healthcare worker and a patient

Setting. Computer laboratory in a university.

Participants. Healthy volunteers.

Main outcome measure. Sensitivity and specificity of automatic detection of the first moment of hand hygiene.

Methods. We captured video and depth images using a Kinect camera and developed computer visual methods to automatically detect the use of alcohol-based hand rub (ABHR), rubbing together of hands, and subsequent contact of the patient by the healthcare worker using depth imagery.
\end{abstract}

Results. We acquired images from 18 different simulated hand hygiene encounters where the healthcare worker complied with the first moment of hand hygiene, and 8 encounters where they did not. The diagnostic accuracy of determining that ABHR was dispensed and that the patient was touched was excellent (sensitivity $100 \%$, specificity $100 \%$ ). The diagnostic 
accuracy of determining that the hands were rubbed together after dispensing ABHR was good (sensitivity $83 \%$, specificity $88 \%$ ).

Conclusions. We have demonstrated that it is possible to automate the direct observation of hand hygiene performance in a simulated clinical setting. We used cheap, widely available consumer technology and depth imagery which potentially increases clinical application and decreases privacy concerns.

\section{KEY WORDS}

Hand hygiene $[\mathrm{MeSH}]$

Image Processing, Computer-Assisted [MeSH]

Cross infection [MeSH]

Quality Assurance, Health Care [MeSH] 


\section{INTRODUCTION}

Healthcare associated infections (HAI) are an important cause of morbidity and mortality in healthcare facilities; $5-15 \%$ of patients admitted to hospital in developed countries will acquire an $\mathrm{HAI}[1,2]$. The problem is even greater in high-risk environments such as intensive care units ( $9-37 \%$ of admissions)[3]. HAIs affect almost 200,000 patients in Australian healthcare facilities and result in approximately 2 million extra hospital bed days annually[4]. Pathogens can be transmitted to susceptible patients by the hands of healthcare workers. Inadequate hand hygiene among healthcare workers was identified as an important cause of HAI by Ignaz Semmelweis in 1846[1] and remains a problem today.

Properly performed hand hygiene effectively reduces HAI[5]. Current World Health Organisation (WHO) and Hand Hygiene Australia guidelines describe the 5 moments of hand hygiene that must be performed[6, 7]. Unfortunately, compliance rates with hand hygiene are frequently low. Hand hygiene compliance rates in Australia across 860 hospitals were estimated to be $82.8 \%$ in June 2015 [8]. Low compliance rates are widespread, and vary between $5 \%$ and $81 \%$ globally[1].

Surveillance of hand hygiene and the collection of quality assurance data are difficult; an ideal method is not available. Direct observation of the 5 moments is currently the most common method for auditing hand hygiene compliance. The WHO Hand Hygiene technical reference manual recommends observing a minimum of 200 opportunities per observation period and per unit of observation (eg. a single ward area) to reliably compare results before and after hand hygiene improvement interventions[6]. Direct observation has major limitations - it is expensive, laborious, and prone to bias. It is subject to an observation bias (Hawthorne effect) where healthcare workers change their behavior whilst being audited, as 
well as other observation and selection biases[1]. Periods of audit are extremely short compared to the breadth of usual clinical care, resulting in gross undersampling. Bias and undersampling are threats to the accuracy of hand hygiene data, and its validity as a performance indicator.

Computer vision is a branch of artificial intelligence that studies how to automatically understand the content of images and video in a human-like manner[9-11]. While computer vision is well established in the area of medical imaging (medical image computing)[12], it is used extremely rarely in clinical medicine where patient (and healthcare worker) privacy is of utmost concern[13]. Concerns about the use of video surveillance in privacy-sensitive environments may be mitigated by the introduction of depth images. Unlike video (RGB) images, depth (or range) images only record the distance of the objects from the camera and do not permit identification of the viewed subjects or to distinguish features beyond outlines. Depth image cameras have become cheap and widely available. We decided to investigate whether computer vision and depth image cameras could be used to surveil hand hygiene in a way that was both clinically feasible and privacy protecting.

\section{Study Objectives.}

1. To demonstrate the feasibility of automated, direct observation and collection of hand hygiene data.

2. To develop computer visual methods capable of reporting compliance with moment 1 (the performance of hand hygiene before touching a patient).

3. To report the diagnostic accuracy of automated, direct observation of moment 1. 


\section{METHODS}

\section{Simulation of the clinical environment and the first moment of hand hygiene.}

We simulated a hospital bedspace in a laboratory at the University of Technology Sydney. Four volunteers performed the roles of patient and healthcare worker, acting as patient and healthcare worker in turn. The camera was placed above the patient's head and pointed toward the foot of the bed. Alcohol-based hand rub (ABHR) was placed on a pedestal at the foot of the bed, near the centre of the camera's frame of view. When the patient was supine, the top of their head was visible to the camera; their face was not. Healthcare workers approached the patient on the bed, with the interaction ending with usual physical examination contact with the patient. Clinically realistic approaches by healthcare workers to the bedside were simulated - this included various combinations with/without dispensing of hand rub, and with/without rubbing of the hands together.

\section{Capture and processing of RGB and depth images.}

The distances from the camera to the bottle, and from the camera to the bed were fixed and measured. We used a Kinect camera (Microsoft Corp) to capture depth images along with RGB images. The depth images bring significant advantages to the automated processing by enabling accurate volumetric scene reconstruction, object tracking, and disambiguation of the occlusions which take place when other objects block the camera view of the targeted objects[14]. Depth images are formed by projecting dots on the scene in the near infrared spectrum and triangulating their distance. To capture the images, we used nuiCapture (v 1.4.0, Cadavid Concepts). The software records synchronous depth and RGB images and automatically extracts the skeleton and face of the tracked subjects from one or multiple Kinect cameras. It also visualises the data using a 3D media player. We exported the files in 
Matlab format, suitable for processing. To automatically detect the hand hygiene events, we used the depth images, and small RGB patches centred on the hand rub.

\section{Determination of compliance with moment 1 of hand hygiene.}

Compliance with moment 1 by a healthcare worker comprises the detection of two events which are expected to take place in the correct order. Event 1 is the use of ABHR which, in our simulations, was placed at the foot of the bed. This event was subdivided into event $1 \mathrm{~A}$ (dispensing of the hand rub) and event 1B (rubbing of hands together vigorously for a minimum amount of time). Event 2 is the subsequent touching of the patient. Event 2, when not preceded by Event 1 was considered non-compliance with moment 1 . The computer vision techniques we used to detect events $1 \mathrm{~A}, 1 \mathrm{~B}$ and 2 are described below.

\section{Computer vision techniques for detection of dispensing ABHR (Event 1A).}

In each frame, we selected a window of pixels centred on the handrub bottle. Dispensing of handrub was inferred if a hand remained in contact with the bottle for a minimum duration (set to 10 frames). Detection consisted of: (i) skin segmentation (detection of the presence of skin-coloured pixels in the pixel window), (ii) counting of skin pixels in close proximity to the hand rub bottle, and (iii) declaring detection if the pixel count was above a given threshold and persisted for a minimum of 10 frames.

\section{Computer vision techniques for detection of hand rubbing (Event 1B)}

This followed only if Event 1A was detected. This detection included (i) detection and removal of the static background scene to highlight the subjects. Detection of the background scene was achieved by running a temporal filter that returned the maximum depth recorded at each pixel location over a period of time (assuming that the background scene would be in view at some point in time); (ii) division of the area of interest (hands) into a grid of 
overlapping windows, and (iii) selection of pixels in each window if they are a) within a given depth range, b) they are segmented as skin and c) they change depth value over time (i.e., are moving objects).

A "hand hypothesis" was then formed if the number of selected pixels was above a threshold. When a hand hypothesis was detected, we used a machine learning classifier (a support vector machine) to detect the rubbing of hands $[15,16]$. The classifier was trained with 600 manually-annotated images, half depicting hand rubbing and half, still hands. Hand rubbing was declared if its occurrence was detected continuously for at least 50 frames.

\section{Computer vision techniques for detection of touching the patient (Event 2).}

This was similar to the method used for Event 1A (dispensing hand rub). The area of interest around the bed/patient was selected, and detection of skin pixels above threshold was used as a proxy for the detection of bed/patient contact by the healthcare worker's hands.

\section{Outcome measures and diagnostic accuracy.}

Automatic detection of hand hygiene events requires machine learning (or training) from a set of manually-annotated data. The learned procedure can then be applied to another set for testing (validation). Cycles of training and testing should be repeated several times and results averaged in order to marginalise the impact of the data set as a random variable in the experiment[17]. For this reason, our experiments have been carried out following an $n$-fold cross validation protocol. The data set was divided into three subsets, A, B, and C, and in each experiment, we have used two joined for training and the third one for testing. This process was repeated three times and the accuracy averaged. 
The gold standard for compliance with moment 1 was direct observation of the RGB images by study personnel. We developed automated computer visual methods to detect 3 events necessary to determine compliance with moment 1 : (1A) dispensing of hand rub by the healthcare worker, (1B) rubbing together of hands by the healthcare worker, and (2) touching the patient. For each of these three events, we measured true positive (TP), false negative (FN), true negative (TN) and false positive (FP) detections. Compliance with moment 1 was defined as the complete performance of events $1 \mathrm{~A}, 1 \mathrm{~B}$, and 2 in the correct order. Violation of moment 1 is defined as the performance of event 2 without preceding performance of events $1 \mathrm{~A}$ and $1 \mathrm{~B}$ in correct order.

\section{Ethics and reporting.}

This project was exempt from the need for ethical review, according to guidelines for quality improvement in our institution[18, 19]. We followed SQUIRE 2.0 reporting guidelines[20]. 


\section{RESULTS}

For the experiments, a total of 26 videos (both depth and colour frames) were acquired. An actor simulating a healthcare worker correctly complied with moment 1 in 18 videos (positive samples), and failed to do so in 8 videos (negative samples). Figure 1 shows typical RGB and depth images from our simulated experiments.

\section{Application of computer vision to hand hygiene observation.}

The use of computer vision to detect the use of ABHR and rubbing together of the hands is shown in Figure 2. The detection of subsequent touching of the patient is summarized in Figure 3.

A side-by-side comparison of video showing a simulated handwashing encounter, and the corresponding, depth imagery which relatively protects privacy is shown in Figure 4.

\section{Diagnostic accuracy of computer vision detection of hand hygiene moment 1.}

The videos acquired consisted of the following true events: 26 samples of Event 1A (18 positives and 8 negatives); 26 samples of Event 1B (18 positives and 8 negatives); and 52 samples of Event 2 (26 positives and 26 negatives, obtained by considering the parts where the clinician was close to the patient and did and did not touch it, respectively). The diagnostic accuracy of detecting the three separate events is reported in Table 1 (TP: true positives, TN: true negatives, FP: false positives, FN: false negatives), and corresponding sensitivity $(\mathrm{TP} /(\mathrm{TP}+\mathrm{FN}))$ and specificity $(\mathrm{TN} /(\mathrm{TN}+\mathrm{FP}))$. Overall, the sensitivity of our methods in correctly detecting compliance with moment 1 was $83 \%$, and the specificity was $88 \%$. 


\section{DISCUSSION}

We have demonstrated the feasibility of auditing hand hygiene using depth imagery and computer vision. Our methods were excellent at detecting the dispensing of hand rub and subsequent manual contact of the patient by the healthcare worker ( $100 \%$ detection). Detection occurred in real time and without the need for video (RGB) images. We used widely available, affordable consumer technology (a Microsoft Kinect camera).

Our findings are significant because HAI and inadequate hand hygiene are a very important public health problem, and the existing strategies for measuring it and managing it are lacking. The bias, under sampling and cost problems of direct observation by human auditors could all potentially be improved by an objective, continuous and inexpensive electronic method such as the one we have described. There is a large Hawthorne effect of auditing on hand hygiene compliance[21]. This can decrease the validity of performance indicator data, but is good for actual hand hygiene practice during periods of audit. Auditing of hand hygiene may be an effective therapeutic intervention for HAI if it can be applied for long periods. We think automated electronic methods are the only way to achieve this.

Technological approaches to improving hand hygiene have been employed before[22]. Remote video auditing with feedback $[23,24]$ is effective but is unlikely to be feasible or affordable on a large scale. Electronic devices can improve training, but are not always effective at improving compliance[25, 26]. Other methods involving sensors on hand rub dispensers, health care workers or both are also relatively expensive and require special equipment[27-31]. Our methods do not require special equipment, do not require transmitters 
or sensors to be applied in the bed area, and are readily deployable anywhere (a single depth image camera is mounted above the head of the bed).

Despite the potential for this approach, our study had important limitations. The clinical setting was simulated and highly controlled: a single healthcare worker approached a supine patient, and used ABHR that was positioned in an elevated position at the foot of the bed. Real clinical care is relatively chaotic, and we have not evaluated these methods in that environment. Our methods were not as accurate at detecting the rubbing together of hands by the healthcare worker ( $83 \%$ true positive rate). Skin segmentation relies on skin coloured pixel detection and is reasonably accurate[32], but untested in clinical areas where non-skin coloured gloves are frequently worn. We believe the use of skeletal data provided by the Kinect camera may potential overcome this problem. We do not know how our methods would perform with multiple healthcare workers in the same area, or with other moments of hand hygiene detection.

We have avoided the substantial ethical and privacy concerns that would arise if electronic surveillance measures were deployed in clinical areas by conducting this work in a laboratory simulation. These concerns would be insurmountable if our methods required the capture (and especially storage) of video (RGB) images. By excluding the patient's face from the field of view, and the exclusive use of non-identifying depth imagery, we believe our methods provide a substantial level of inherent privacy protection. Further development and deployment in clinical areas would need to be conducted with great care and sensitivity[13, 33].

In conclusion, the potential for clinical application is significant. No video imagery needs to be stored (or even captured). The equipment needed is widely available and can be deployed 
anywhere. It could be paired with real-time feedback to healthcare workers to encourage ABHR use prior to touching their patient. It could generate continuous auditing data for use by managers in real time, or provide aggregate reports whilst avoiding identification or video surveillance of staff. The next logical step would be to evaluate these methods in a real clinical area using volunteers instead of patients. The technology should only be applied widely outside research settings if it is known to reduce HAI, raises no significant privacy concerns, is affordable, and robust.

\section{ACKNOWLEDGEMENTS}

Funding - No Funding

Conflict of interest statement - All authors declare that they have no conflicts of interest. 


\section{REFERENCES}

1. World Health Organisation (WHO). WHO Guidelines on Hand Hygiene in Health Care: a summary 2009 [updated Accessed 1/8/2015.1/8/2015]. Available from: http://www.who.int/gpsc/5may/tools/who_guidelines-handhygiene_summary.pdf.

2. Spelman DW. Hospital-acquired infections. The Medical journal of Australia. 2002;176(6):286-91.

3. Vincent JL. Nosocomial infections in adult intensive-care units. Lancet (London, England). 2003;361(9374):2068-77.

4. Australian Commission on Safety and Quality in Health Care. Cruickshank M, Ferguson J, editors. Reducing Harm to Patients from Health Care Associated Infection: The Role of Surveillance. 2008 [updated Accessed on 1 Sep 2016. Available from: http://www.safetyandquality.gov.au/wp-content/uploads/2008/01/Reducing-Harm-to-PatientRole-of-Surveillance1.pdf.

5. Bernard S. Refractory Out-Of-Hospital Cardiac Arrest Treated With Mechanical CPR, Hypothermia, ECMO and Early Reperfusion. NCT01186614 Australian New Zealand Clinical Trials Registry 2010 [Available from:

https://www.anzctr.org.au/Trial/Registration/TrialReview.aspx?id=2876\&isClinicalTrial=Tru e.

6. World Health Organisation (2009). Hand hygiene technical reference manual: to be used by health-care workers, trainers and observers of hand hygiene practices [updated Accessed on 1 Aug 2015. Available from: http://apps.who.int/iris/bitstream/10665/44196/1/9789241598606_eng.pdf.

7. Hand Hygiene Australia. 5 Moments for Hand Hygiene [updated Accessed 1 Sep 2016. Available from: http://www.hha.org.au/home/5-moments-for-hand-hygiene.aspx.

\section{Do A, Cretikos, M, Muscatello, D, Rose, N. Epidemiology} of out-of-hospital cardiac arrests, NSW, 2012: Time, place and person. Sydney: Centre for Epidemiology and Evidence, NSW Ministry of Health; 2013.

9. Haralick RM, Shapiro LG. Glossary of computer vision terms. Pattern Recognition. 1991;24(1):69-93.

10. Fisher RB, Breckon TP, Dawson-Howe K, Fitzgibbon A, Robertson C, Trucco E, et al. Dictionary of Computer Vision and Image Processing: John Wiley \& Sons, Ltd; 2016. 324-72 p. 
11. Shah M. Fundamentals of Computer Vision. 1997 [updated Accessed 1 Sep 2015. Available from: http://crcv.ucf.edu/gauss/BOOK.PDF.

12. Handels H, Deserno TM, Meinzer HP, Tolxdorff T. Image Analysis and Modeling in Medical Image Computing. Recent Developments and Advances. Methods of Information in Medicine. 2012;51(5):395-7.

13. Palmore TN, Henderson DK. Big Brother is Washing...Video Surveillance for Hand Hygiene Adherence, Through the Lenses of Efficacy and Privacy. Clinical Infectious Diseases. 2012;54(1):8-9.

14. Jana A. Kinect for Windows SDK Programming Guide: Packt Publishing; December 2012.

15. Cortes C, Vapnik V. Support-vector networks. Machine Learning. 1995;20(3):273-97. 16. Avalli L, Maggioni E, Formica F, Redaelli G, Migliari M, Scanziani M, et al. Favourable survival of in-hospital compared to out-of-hospital refractory cardiac arrest patients treated with extracorporeal membrane oxygenation: an Italian tertiary care centre experience. Resuscitation. 2012;83(5):579-83.

17. Dietterich TG. Approximate Statistical Tests for Comparing Supervised Classification Learning Algorithms. Neural Computation. 1998;10(7):1895-923.

18. NSW Health: Office for Health and Medical Research. Human Research Ethics Committees - Quality Improvement \& Ethical Review: A Practice Guide for NSW (2007, GL2007_020) [updated Accessed 29 Aug 2016. Available from: http://www0.health.nsw.gov.au/policies/g1/2007/GL2007_020.html.

19. BMJ Quality \& Safety. Policy on ethics review for quality improvement reports Updated April 2014. [updated Accessed 29 Aug 2016. Available from: http://qualitysafety.bmj.com/site/misc/PolicyonEthicReviews.pdf.

20. Ogrinc G, Davies L, Goodman D, Batalden P, Davidoff F, Stevens D. SQUIRE 2.0 (Standards for QUality Improvement Reporting Excellence): revised publication guidelines from a detailed consensus process. BMJ Quality \& Safety. 2015.

21. Hagel S, Reischke J, Kesselmeier M, Winning J, Gastmeier P, Brunkhorst FM, et al. Quantifying the Hawthorne Effect in Hand Hygiene Compliance Through Comparing Direct Observation With Automated Hand Hygiene Monitoring. Infection Control \& Hospital Epidemiology. 2015;36(8):957-62.

22. Srigley JA, Gardam M, Fernie G, Lightfoot D, Lebovic G, Muller MP. Hand hygiene monitoring technology: a systematic review of efficacy. Journal of Hospital Infection. 2015;89(1):51-60. 
23. Armellino D, Hussain E, Schilling ME, Senicola W, Eichorn A, Dlugacz Y, et al. Using High-Technology to Enforce Low-Technology Safety Measures: The Use of Thirdparty Remote Video Auditing and Real-time Feedback in Healthcare. Clinical Infectious Diseases. 2012;54(1):1-7.

24. Armellino D, Trivedi M, Law I, Singh N, Schilling ME, Hussain E, et al. Replicating changes in hand hygiene in a surgical intensive care unit with remote video auditing and feedback. American journal of infection control. 2013;41(10):925-7.

25. Kwok YL, Callard M, McLaws ML. An automated hand hygiene training system improves hand hygiene technique but not compliance. American journal of infection control. 2015;43(8):821-5.

26. Higgins A, Hannan MM. Improved hand hygiene technique and compliance in healthcare workers using gaming technology. The Journal of hospital infection. 2013;84(1):32-7.

27. Fisher DA, Seetoh T, May-Lin HO, Viswanathan S, Toh Y, Yin WC, et al. Automated Measures of Hand Hygiene Compliance among Healthcare Workers Using Ultrasound: Validation and a Randomized Controlled Trial. Infection Control \& Hospital Epidemiology. 2015;34(9):919-28.

28. Levchenko AI, Boscart VM, Fernie GR. The effect of automated monitoring and realtime prompting on nurses' hand hygiene performance. Computers, informatics, nursing : CIN. 2013;31(10):498-504.

29. Marra AR, D'Arco C, Bravim Bde A, Martino MD, Correa L, Silva CV, et al. Controlled trial measuring the effect of a feedback intervention on hand hygiene compliance in a step-down unit. Infection control and hospital epidemiology. 2008;29(8):730-5.

30. Swoboda SM, Earsing K, Strauss K, Lane S, Lipsett PA. Electronic monitoring and voice prompts improve hand hygiene and decrease nosocomial infections in an intermediate care unit. Critical care medicine. 2004;32(2):358-63.

31. Sahud AG, Bhanot N, Radhakrishnan A, Bajwa R, Manyam H, Post JC. An electronic hand hygiene surveillance device: a pilot study exploring surrogate markers for hand hygiene compliance. Infection control and hospital epidemiology. 2010;31(6):634-9.

32. Jones MJ, Rehg JM. Statistical Color Models with Application to Skin Detection. International Journal of Computer Vision. 2002;46(1):81-96.

33. Tim Lahey. A Watchful Eye in Hospitals. New York Times. 16 Feb 2014. 


\section{TABLES AND FIGURE LEGENDS}

\section{Table 1}

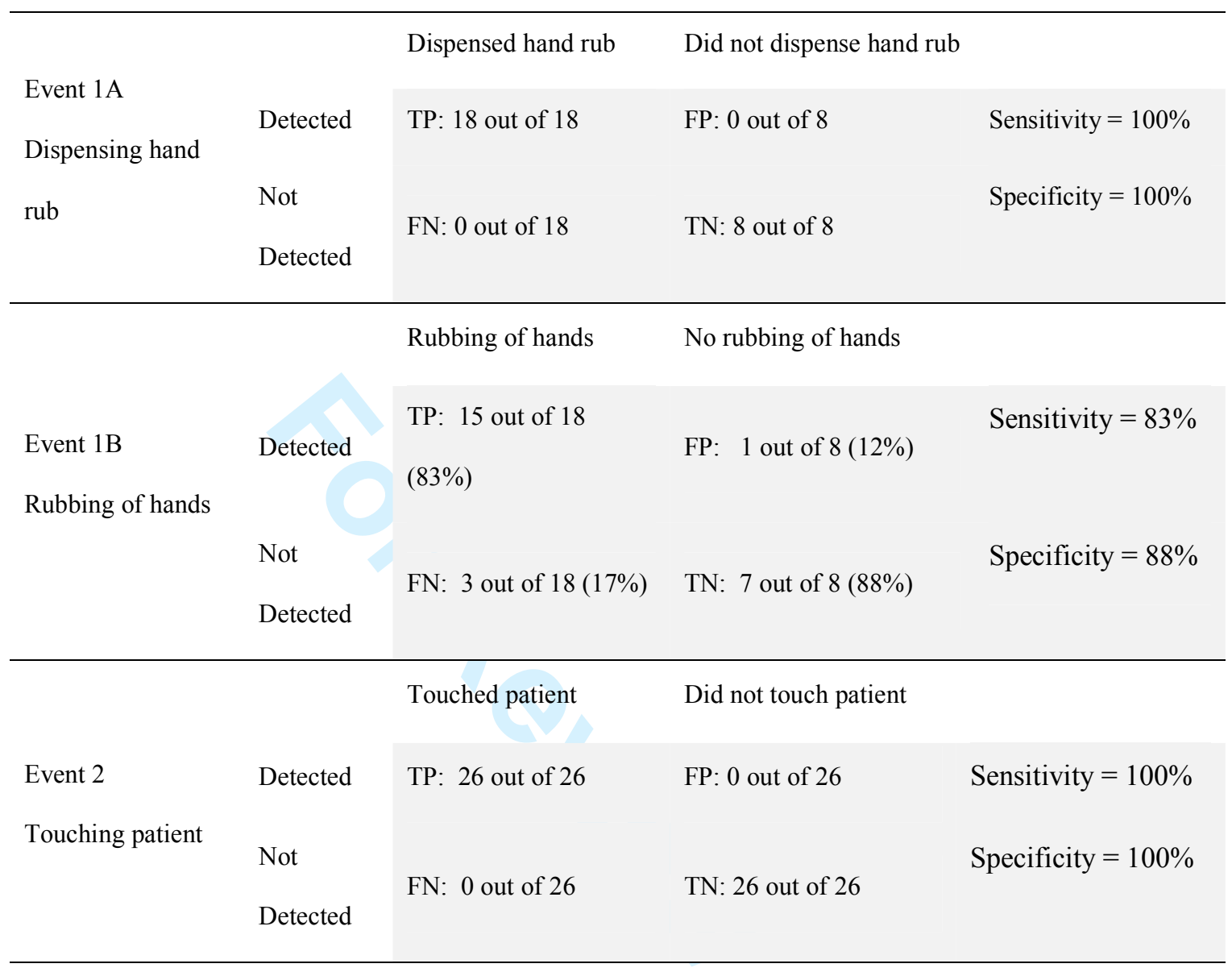

Table 1. Diagnostic accuracy of computer visual detection of three events comprising the first moment of hand hygiene. $\mathrm{TP}=$ true positive, $\mathrm{FN}=$ false negative, $\mathrm{FP}=$ false positive, $\mathrm{TN}=$ true negative. 


\section{Figure Legends}

Figure 1

An example of the images that were used in this work. Left: video (RGB) image of actual scene. Right: processed depth imagery of the same scene.

Figure 2

The use of computer vision to detect the use of alcohol-based hand rub (Event 1) Top left: video image of the scene. Top centre: the depth frame. Top right: the skeleton extracted from the depth frame, clearly showing the detected position of the hands. Middle: Event 1A: detection of hand rub use. Bottom: Event 1B: sustained rubbing of hands.

Figure 3

The use of computer vision to detect contact between the healthcare worker and patient (Event 2).

Figure 4 (video file)

Side-by-side video and depth images showing a simulated first moment of hand hygiene (use of alcohol-based hand rub followed by touching the patient). 
Figure 1. An example of the images that were used in this work. Left: video (RGB) image of actual scene. Right: processed depth imagery of the same scene.

\section{$211 \times 77 \mathrm{~mm}(96 \times 96 \mathrm{DPI})$}


Figure 2: Top left: depiction of the scene. Top centre: the depth frame. Top right: the skeleton extracted from the depth frame, clearly showing the detected position of the hands. Middle: Event 1A: detection of hand rub use. Bottom: Event 1B: sustained rubbing of hands.

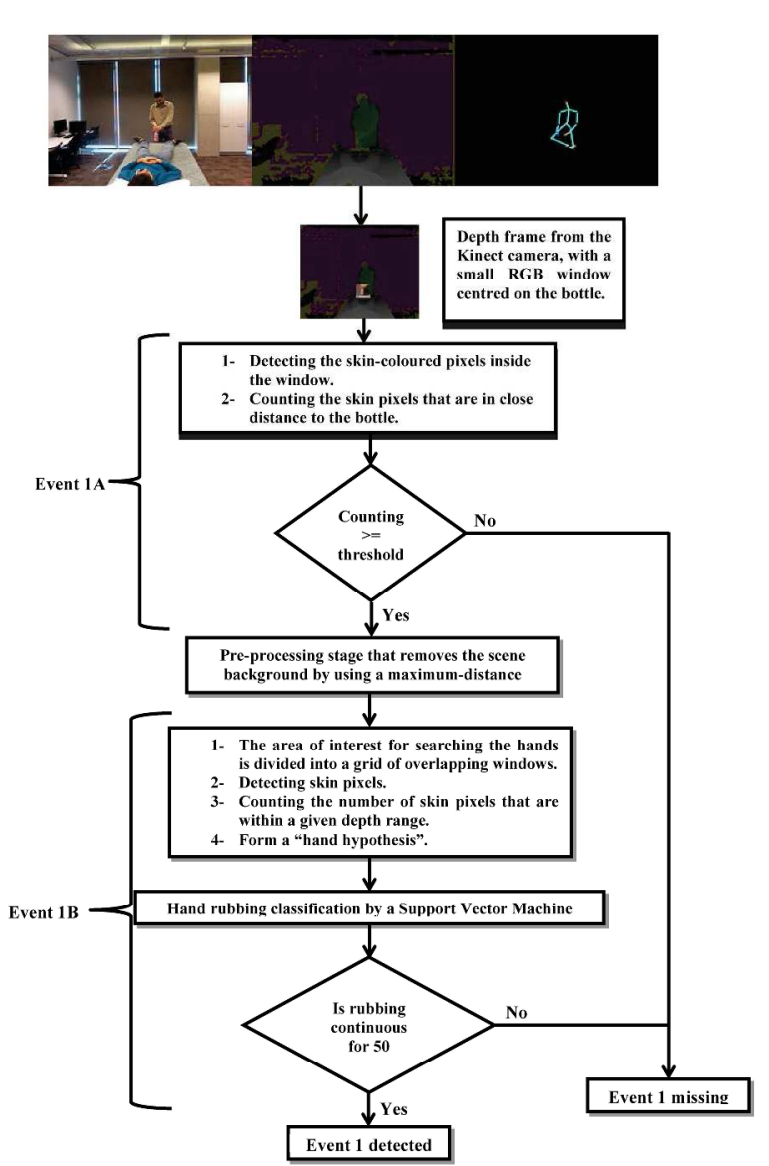

Figure 2. The use of computer vision to detect the use of alcohol-based hand rub (Event 1) Top left: video image of the scene. Top centre: the depth frame. Top right: the skeleton extracted from the depth frame, clearly showing the detected position of the hands. Middle: Event 1A: detection of hand rub use. Bottom: Event 1B: sustained rubbing of hands.

$$
297 \times 420 \mathrm{~mm} \text { ( } 300 \times 300 \text { DPI) }
$$


Figure 3: Event 2: contact with the patient.

Figure 3. The use of computer vision to detect contact between the healthcare worker and patient (Event 2). $297 \times 420 \mathrm{~mm}(300 \times 300$ DPI $)$

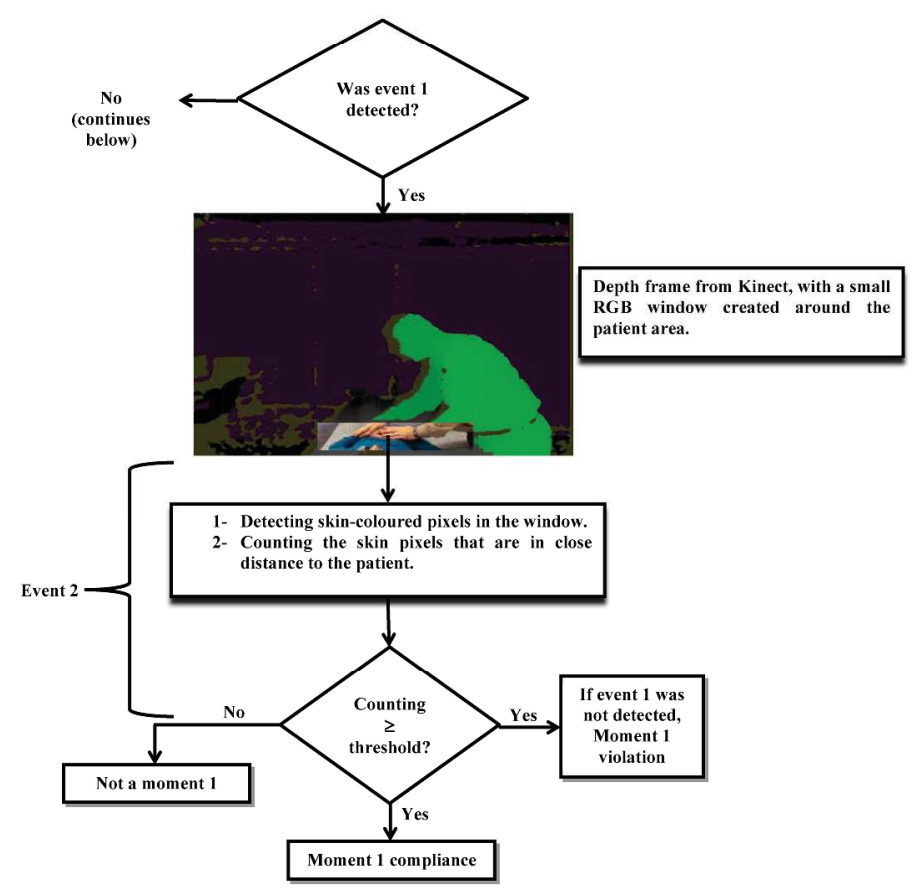

